\title{
Pancreaticoduodenectomy for ductal adenocarcinoma of the pancreatic head with venous resection
}

\author{
Vojko Flis ${ }^{1}$, Stojan Potrc ${ }^{2}$, Nina Kobilica ${ }^{1}$, Arpad Ivanecz ${ }^{2}$ \\ ${ }^{1}$ Department of Vascular Surgery, Surgical clinics, University Clinical Centre Maribor, Slovenia \\ ${ }^{2}$ Department of Abdominal Surgery, Surgical clinics, University Clinical Centre Maribor, Slovenia
}

Radiol Oncol 2016; 50(3): 321-328.

Received 17 December 2014

Accepted 26 March 2015

Correspondence to: Vojko Flis, M.D., Ph.D., Department of Vascular Surgery, Surgical Clinics, University Clinical Centre Maribor, Slovenia. E-mail: vojko.flis@ukc-mb.si

Disclosure: No potential conflicts of interest were disclosed.

Background. Recent reports have shown that patients with vascular tumour invasion who undergo concurrent vascular resection can achieve long-term survival rates equivalent to those without vascular involvement requiring pancreaticoduodenectomy alone. There is no consensus about which patients benefit from the portal-superior mesenteric vein resection and there is no consensus about the best surgical technique of vessel reconstruction (resection with or without graft reconstruction). As published series are small the aim of this study was to evaluate our experience in pancreatectomies with en bloc vascular resection and reconstruction of vessels.

Methods. Review of database at University Clinical Centre Maribor identified 133 patients (average age $65.4 \pm$ 8.6 years, 69 female patients) who underwent pancreatoduodenectomy between January 2006 and August 2014. Clinical data, operative results, pathological findings and postoperative outcomes were collected prospectively and analyzed. Current literature and our experience in pancreatectomies with en bloc vascular resection and reconstruction of portal vein are reviewed.

Results. Twenty-two patients out of 133 (16.5\%) had portal vein-superior mesenteric vein resection and portal vein reconstruction (PVR) during pancreaticoduodenectomy. In fourteen patients portal vein was reconstructed without the use of synthetic vascular graft. In these series two types of venous reconstruction were performed. When tumour involvement was limited to the superior mesenteric vein (SPV) or portal vein (PV) such that the splenic vein could be preserved, and vessels could be approximated without tension a primary end-to-end anastomosis was performed. When tumour involved the SMV-splenic vein confluence, splenic vein ligation was necessary. In the remaining eight procedures interposition graft was needed. Dacron grafts with $10 \mathrm{~mm}$ diameter were used. There was no infection after dacron grafting. One patient had portal vein thrombosis after surgery: it was thrombosis after primary reconstruction. There were no thromboses in patients with synthetic graft interposition. There were no significant differences in postoperative morbidity, mortality or grades of complication between groups of patients with or without a PVR. Median survival time in months was in a group with vein resection 16.13 months and in a group without vein resection 15.17 months. Five year survival in the group without vein resection was $19.5 \%$. Comparison of survival curves showed equal hazard rates with log-rank $p=0.090$.

Conclusions. Survival of patients with pancreatic cancer who undergo an RO resection with reconstruction was comparable to those who have a standard pancreaticoduodenectomy with no added mortality or morbidity. Synthetic graft appeared to be an effective and safe option as an interposition graft for portomesenteric venous reconstruction after pancreaticoduodenectomy.

Key words: pancreaticoduodenectomy; pancreatic cancer; vein resection 


\section{Introduction}

In cases of pancreatic cancer pancreaticoduodenectomy with complete resection offers the only chance for cure. Historically, involvement of regional vascular structures by pancreatic carcinoma has been considered a contraindication for reconstruction. ${ }^{1}$ At the time of diagnosis more than three quarters of patients have locally advanced disease or distant metastasis that preclude radical surgery and 5-year survival after "curative" surgery ranges from 10 to $20 \%$ even in recent large series. ${ }^{2}$ Advances in surgical techniques, perioperative care and the institution of tertiary specialized centres have been the key for a substantial improvement in mortality and morbidity rate. Venous resection (VR) is performed to achieve negative resection margins because the tumour involves the vessel or inflammatory adhesions preclude a safe separation of the vein. Another theoretical benefit of VR is to achieve clearance of surrounding perivascular and perineural tissue. Venous resections (VR) include excision of portal vein (PV), superior mesenteric vein (SMV) or the superior mesenteric-portal vein confluence (SMPV). ${ }^{2}$

Although the utility of aggressive vascular resection in pancreatic adenocarcinoma continues to be debated ${ }^{3,4}$ several institutional series have demonstrated the feasibility of margin negative resection with acceptable morbidity rates comparable to those after isolated pancreaticoduodenectomy. Recent reports also have shown that patients with vascular tumour invasion who undergo concurrent vascular resection can achieve long-term survival rates equivalent to those without vascular involvement requiring PD alone. ${ }^{5-9}$

Reconstruction of the PV or SMV is a challenge for the vascular surgeon because of the lack of size-matched autogenous conduit. In addition, concerns about graft infection have restricted the use of prosthetic grafts during the intra-abdominal surgery. ${ }^{9}$ Numerous techniques of VR have been described, ranging from partial excision of the lateral wall to major segmental resections. ${ }^{10-12}$ The resultant defects can be repaired with either a primary anastomosis or a graft. A variety of different native vessels and synthetic grafts have been described to bridge the defect. Each method, however, has limitations and the optimal conduit and surgical methods remain a controversy. ${ }^{13-15}$ As published series are small the aim of this study was to evaluate our experience in pancreatectomies for ductal adenocarcinoma with en bloc vascular resection and reconstruction of vessels.

\section{Methods}

Approval of the Research Council of Surgical Clinics was obtained to perform the audit of patients with pancreatic adenocarcinoma undergoing surgery between January 2006 and August 2014. Clinical data, operative results, pathological findings and postoperative outcomes were collected prospectively and analyzed.

\section{Preoperative evaluation}

All patients underwent contrast-enhanced CT as a routine preoperative work-up. Magnetic resonance imaging, endoscopic ultrasound scan, and laparoscopy were performed on an individual basis based on the multidisciplinary team discussion. The final operative decision lay with the surgeon at laparatomy. Only patients deemed respectable preoperatively were included. The criteria for en bloc resection where there was no evidence of metastatic disease were the following: tumour not involving the root of the small bowel mesentery; tumour not involving the superior mesenteric artery, celiac axis, or hepatic artery; and intention of obtaining R0 resection margin status. Patients with portal vein occlusion were not included.

The general condition of the patients was determined by American Society of Anesthesiologists (ASA) score. ${ }^{16}$ For study purposes regarding preoperative level of bilirubin, Carcinoembryonic antigen (CEA) and carbohydrate antigen 19-9 (CA 199) subgroups were formed.

\section{Perioperative data}

The operative approach was a median laparotomy until the 2007 later a bilateral subcostal laparotomy was preferred. Ultrasound examination of the pancreas was always used for evaluation of vascular involvement and for possible liver metastases.

Pancreatic head resections were done in a conventional manner. ${ }^{17}$ In the last two years the artery first approach (posterior approach) was used whenever the infiltration and resection of superior mesenteric vein were planned. ${ }^{18}$ Jejunum was exclusively used for the anastomosis to the pancreas (duct to mucosa type, two layers) and for bile reconstruction (on layer) successively. A separate Roux en Y loop for pancreatojejuno anastomosis was done only in 2 patients. All gastro/pyloro-jejuno anastomoses were placed above the colon. In all patients with pylorus-preserving pancreaticoduodenectomy (PPPD)/Whipple resection a small en- 
teroentero anastomosis was added to connect the afferent end efferent loop of gastro/pyloro-jejuno anastomosis. In total and left pancreatectomy by rule the spleen and the splenic vessels were resected "en block", however in three 3 total pancratectomies with additional intraductal papillary mucinous neoplasia of the left pancreas the spleen was preserved. The pancreatic stump was almost exclusively closed with sutures. At the end of operation abdominal drains were always placed. In 3 cases spleen could be preserved, however in others ligation of splenic artery at the origin and splenic vein at the confluence was done. For prevention of pancreatic fistula in cases with the soft texture of the pancreas somatostatin $0.6 \mathrm{mg}$ daily for 5 to 8 days was administrated. ${ }^{19}$

Vascular resections were carried out as primary closure of the vein, end to end anastomosis, or a segmental resection and reconstruction with interposition graft. Dacron grafts with $10 \mathrm{~mm}$ diameter were used.

\section{Postoperative follow up}

After surgery, the patients were followed up to detect complications, local recurrence, distant metastasis and survival rate. The surgical complications were noted and classified. ${ }^{20}$ Laboratory tests and control of the tumour markers CEA and CA 19-9 as well as ultrasound and/or CT scans were obtained at three to four month intervals within 2 years after the operation and then later at six month intervals. The samples of fluid on drains were regularly examined for amylase on the day 4 and anytime in the course if the volume on drains was more than $50 \mathrm{ml}$ to rule out the possible pancreatic fistula (PF). ${ }^{21}$

Adjuvant chemotherapy was given according to final patohistological stage (pTNM) and was gemcitabine based on the majority of cases.

Hospital stay was defined as time from operation to final dismissal from the hospital.

30 and 60 day mortality was defined as any postoperative death within 30 or 60 days after the operation.

All resected specimens were sent to standardized pathohistological work up to the Department of pathology in Maribor.

\section{Statistical analysis}

Perioperative and clinicopathological parameters were evaluated and further compared between the two groups of patients. Categorical data were compared using $x^{2}$ test or Fischer's exact test.
TABLE 1. Selected clinical characteristics and preoperative data in patients undergoing pancreaticoduodenectomy with or without vein resection (VR). There is no statistically important differences between both groups

\begin{tabular}{|c|c|c|c|}
\hline Variable & $\begin{array}{l}\text { Without VR } \\
(n=111)\end{array}$ & $\begin{array}{l}\text { With VR } \\
(n=22)\end{array}$ & P-value test \\
\hline \multicolumn{4}{|l|}{ Demographics } \\
\hline Age (average years) & $65.6 \pm 7.7$ & $63.95 \pm 9.5$ & $P=0.45 ; \dagger$ test \\
\hline Sex (male:female) & $53: 58$ & $9: 13$ & $P=0.64 ;$ Chi square \\
\hline \multicolumn{4}{|l|}{ ASA score } \\
\hline 1 & 34 & 7 & \\
\hline 2 & 61 & 12 & \\
\hline 3 & 16 & 2 & \\
\hline 4 & 0 & 0 & $P=0.83 ;$ Chi square \\
\hline \multicolumn{4}{|l|}{ Bilirubin level } \\
\hline Below 100mmol/l & 77 & 18 & \\
\hline Above $100 \mathrm{mmol} / /$ & 34 & 4 & $P=0.306 ;$ Chi square \\
\hline \multicolumn{4}{|l|}{ CEA (ng/l) } \\
\hline Increased (> 5ng/l) & 30 & 5 & $P=0.795 ;$ Chi square \\
\hline \multicolumn{4}{|l|}{ CA $19-9$ (IU/I) } \\
\hline Increased (> 30IU/I) & 78 & 16 & $P=1.0 ;$ Chi square \\
\hline
\end{tabular}

$\mathrm{ASA}=$ American Society of Anesthesiologists; $\mathrm{CA} 19-9=$ carbohydrate antigen 19-9; $\mathrm{CEA}=$ carcinoembryonic antigen

Comparison of two different means was done by t-test. Survival curves were computed according to the Kaplan-Meier method. SPSS version 20 software (SPSS, IBM Corp, Armonk, NY, USA) was used to collect data and perform statistical analyses.

\section{Results}

Review of database at University Clinical Centre Maribor identified 133 patients (average age $65.4 \pm$ 8.6 years, 69 female patients) who underwent pancreaticoduodenectomy for ductal adenocarcinoma of the pancreatic head between January 2006 and August 2014.

In the first group there were one 111 patients (83.5\%), (53 male, 58 female patients, average 65.6 \pm 7.7 years) with a standard pancreaticoduodenectomy without portal vein resection (PD - VR). In the second group there were 22 patients out of 133 (16.5\%) (10 male, 12 female patients, average 63.95 \pm 9.5 years) who had portal vein - superior mesenteric vein resection and portal vein reconstruction (PD + VR) during pancreaticoduodenectomy. There was no statistically important difference in preoperative patient characteristics between the PD - VR and PD + VR groups (Table 1). The dif- 
TABLE 2. TNM stage in 111 patients who underwent pancreaticoduodenectomy without vein resection in comparison with vein resection (VR) group. There is no statistically important difference in NO and $\mathrm{N} 1$ stage between both groups $(P=0.432$, Fischer's exact test, no statistical significance).

\begin{tabular}{ccc}
\hline TNM stage & Without VR $(n=111)$ & With VR $(n=22)$ \\
\hline T0 & 0 & 0 \\
T1 & 10 & 0 \\
T2 & 26 & 0 \\
T3 & 75 & 22 \\
T4 & 0 & 0 \\
N0 & 32 & 4 \\
N1 & 79 & 18
\end{tabular}

TABLE 3. List of surgical complications developed after pancreatoduodenectomy. Comparison between group without vein resection (PD [pacreaticoduodenectomy] - VR [vein resection]) and group with vein resection (PD + VR)

\begin{tabular}{lcc}
\hline Type of surgical complication & $\begin{array}{c}\text { Without VR } \\
(\mathbf{n}=111)\end{array}$ & $\begin{array}{c}\text { With VR } \\
(\mathbf{n}=22)\end{array}$ \\
\hline Pancreatic fistula & $5(4.5 \%)$ & $1(4.5 \%)$ \\
Bile leak & $3(2.7 \%)$ & $1(4.5 \%)$ \\
Intraperitoneal bleeding & $6(5.4 \%)$ & 0 \\
Abdominal abscess & $5(4.5 \%)$ & 0 \\
Gastric emptying syndrome & $1(0.9 \%)$ & $1(4.5 \%)$ \\
Rupture of the laparatomy & $4(3.6 \%)$ & 0 \\
Necrosing pancreatitis & $1(0.9 \%)$ & 0 \\
Ileus of Roux-Y & $1(0.9 \%)$ & 0 \\
Critical ischemia of the colon & $1(0.9 \%)$ & 0 \\
Poral vein thrombosis & 0 & $1(4.5 \%)$ \\
60 day mortality & $5(4.5 \%)$ & $1(4.5 \%)$ \\
\hline
\end{tabular}

ference between groups in TNM staging is shown in Table 2. All patients in PD + VR group are in stage T3, however, there was no difference in $\mathrm{N}$ and $\mathrm{M}$ classification $(\mathrm{P}=0.432$, Fischer's exact test, no statistical significance) (Table 2). Surgical complications are listed in Table 3. The occurrence of surgical complications in the second group was to low for valid statistical comparison. There was no statistically important difference in histology (extension) of resection margins between groups (Table 4), (Chi-square 2.79, $\mathrm{p}=0.247$ ). There was one early death in vein reconstruction group (4.5\%) and 5 deaths in another group (4.5\%). There was no statistically significant difference between both
TABLE 4. Resection margins of extirpated tumors. There is no statistically important difference bewteen both groups (with or without venous resection [VR]) (Chi-square 2.79, $p=0.247$ )

\begin{tabular}{ccc}
\hline $\begin{array}{c}\text { Resection } \\
\text { margin }\end{array}$ & Without VR $(\mathbf{n}=111)$ & With VR $(\mathbf{n}=\mathbf{2 2})$ \\
\hline RO & 102 & 18 \\
R0,1 & 5 & 3 \\
R1 & 4 & 1 \\
\hline
\end{tabular}

TABLE 5. Cause of intrahospital deaths between both groups. $(P=1.0$, Fischer's exact test, no statistical significance in death rate)

\begin{tabular}{lcc}
\hline Cause of death & Without VR & With VR \\
\hline $\begin{array}{l}\text { Massive pulmonary } \\
\text { embolia }\end{array}$ & 1 & 0 \\
$\begin{array}{l}\text { Cerebrovascular insult } \\
\text { Myocardial infarction }\end{array}$ & 1 & 0 \\
Bronchopneumonia & 2 & 1 \\
\hline
\end{tabular}

$V R=$ vein resection

groups ( $\mathrm{P}=1.0$, Fischer's exact test, no statistical significance in death rate). Causes of intrahospital deaths are listed in Table 5.

Adjuvant chemotherapy didn't impact the long term survival. In fourteen patients portal vein was reconstructed without the use of synthetic vascular graft. In these series two types of venous reconstruction were performed. When tumour involvement was limited to the superior mesenteric vein (SPV) or portal vein (PV) such that the splenic vein could be preserved, and vessels could be approximated without tension a primary end-to-end anastomosis was performed. When tumour involved the SMV - splenic vein confluence, splenic vein ligation was necessary (Figure 1). In the remaining eight procedures interposition graft was needed. Dacron grafts with $10 \mathrm{~mm}$ diameter were used. There was no infection after dacron grafting. One patient had portal vein thrombosis after surgery: it was thrombosis after primary reconstruction. There were no thromboses in patients with synthetic graft interposition.

\section{Survival analysis}

Median survival time in months was in a group with vein resection (PD + VR) 16.1 months and in a group without vein resection (PD - VR) 15.2 months. Five year survival in the group without 
vein resection was $19.5 \%$. Comparison of survival curves showed equal hazard rates with log-rank $p$ $=0.090(\mathrm{z}=1.659$ at $5 \% \mathrm{C} ; \mathrm{C}=1.96)$ (Figure 2 and Figure 3).

\section{Discussion}

Pancreatic cancer is the $4^{\text {th }}$ most common cause of cancer death in the Western world. ${ }^{22}$ The mortality rate closely approximates the incidence, but surgical resection is generally accepted as having a beneficial effect on survival. ${ }^{1,2}$ However, due to the presence of metastatic disease or invasion of local structures, most patients are not operative candidates at presentation. Historically, involvement of regional vasculature by pancreatic carcinoma has been considered a contraindication to resection. ${ }^{1}$ Advances in surgical technique, intensive care and neoadjuvant chemotherapy have increased the rate of resectability, particularly for patients whose pancreatic cancer involves the portal vein (PV) and superior mesenteric vein (SMV). Vascular resection has become routine for locally advanced pancreatic tumours. Venous resections are supported only when an R0 resection is achieved. Many recent studies have shown that venous resection does not alter overall mortality and is therefore not a contraindication to extended tumour resection. ${ }^{1,15}$ However the resection and reconstruction of the $\mathrm{PV}$ is a technically challenging procedure and the number of patients undergoing this type of operation in any given series is small. ${ }^{14,15}$ Currently, venous resection has been reported in up to $20 \%$ of pancreaticoduodenectomies at high-volume pancreatic surgery centers. ${ }^{12,22}$ It has been suggested that pancreatic head resection (PHR) with venous resection (VR) might be associated with a higher complication rate when compared with pancreatic head resection alone. ${ }^{14}$ In our study as in some other studies the morbidity of PHR combined with VR was similar to PHR alone. ${ }^{22}$ In the meta-analysis by Zhou et al., of 19 studies that reported on mortality, no difference was observed between PHR with VR and PHR alone. ${ }^{23}$

Arterial resection is more rarely performed; they can include the celiac trunk, superior mesenteric and hepatic arteries, but usually arterial involvement is regarded as a contraindication to surgery as it carries a higher postoperative mortality, lack of survival benefit and are more likely associated to $\mathrm{R} 1$ resections. ${ }^{12}$

Some studies show a greater proportion of R1 resections in pancreatectomies with vein resection

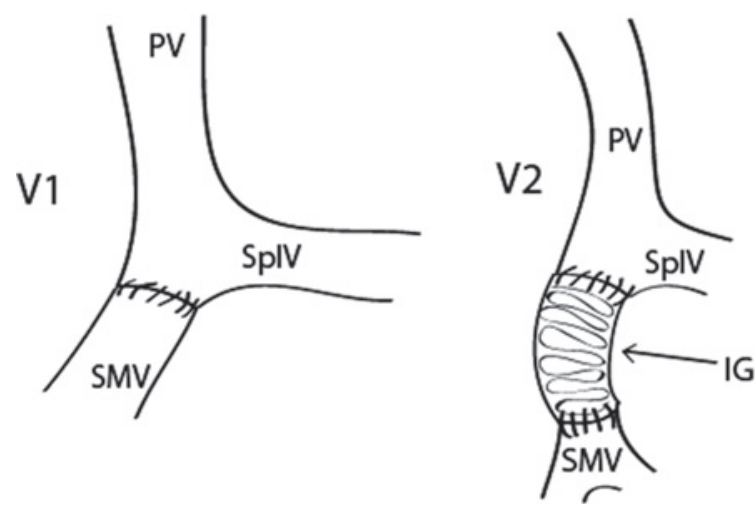

FIGURE 1. In presented series basically two types of venous reconstruction were performed. When tumour involvement was limited to the superior mesenteric vein (SMV) or portal vein (PV) such that the splenic vein (SpIV) could be preserved, and vessels could be approximated without tension a primary end-to-end anastomosis was performed (V1). In the remaining cases interposition graft (IG) was needed (V2).

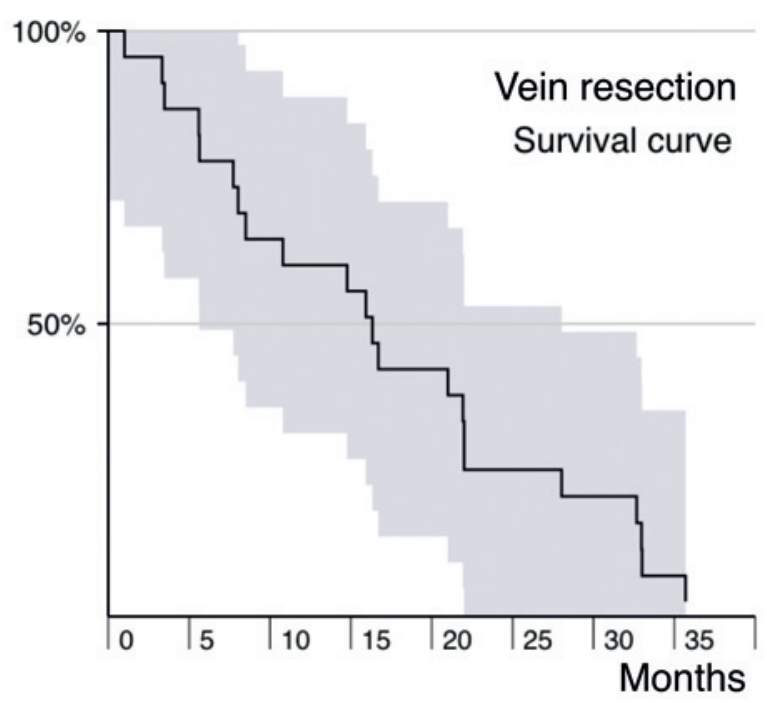

FIGURE 2. Kaplan-Meier survival plot for patients with vein reconstruction (pacreaticoduodenectomy [PD]+ vein resection [VR]). Median survival time in months was in this group 16.1 months.

than in pancreatectomies alone. ${ }^{22}$ However, the greater proportion of $\mathrm{R} 1$ resections in PHR with VR group might be connected with differences in histopahtological reporting. The nature of tissue sampling of the circumferential resection margins differs between institutions. As a result, R1 rates vary considerably in the literature ranging from $37 \%$ to $75 \% .^{22,24}$ Additionally, some studies have shown that R1 resections have had no adverse effect on survival. ${ }^{12,22}$ In contrast, the ESPAC-1 trial suggested that resection margin status was a nega- 


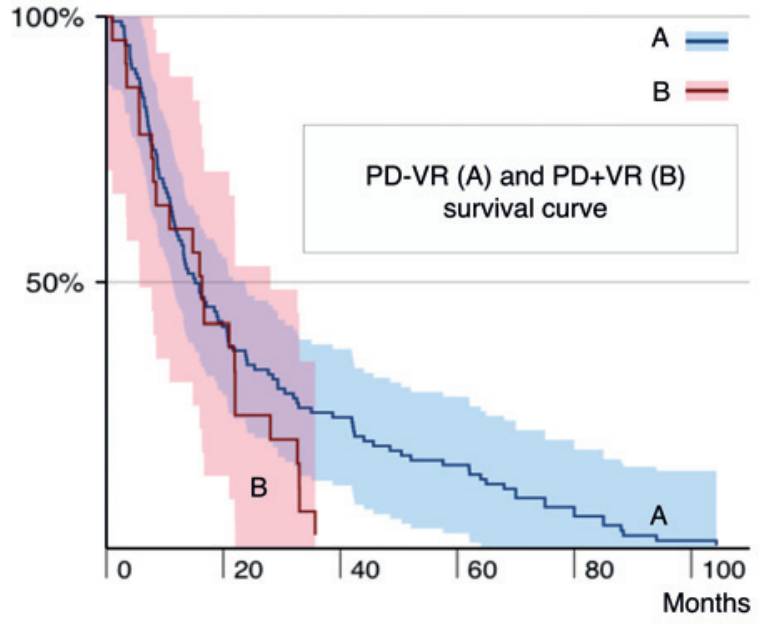

FIGURE 3. Comparison of Kaplan-Meier survival plots for both groups. Median survival time in months was in group with vein resection (pacreaticoduodenectomy [PD]+ vein resection [VR]) 16.1 months (line B) and in group without vein resection (PD - VR) 15.2 months (line A). Five year survival in group without vein resection (line B) was $19.5 \%$. Comparison of survival curves showed equal hazard rates with log-rank $p=0.090(z=1.659$ at $5 \% \mathrm{C} ; \mathrm{C}=1.96)$.

tive predictor of survival. ${ }^{25}$ In our study R1 status had no adverse effect on survival. However, with such discrepancies in the literature with regard to the resection margin status it could be postulated that until histopathologic reporting is more standardized universally its role as a prognostic indicator remains equivocal. ${ }^{22}$ The opponents of the PHR with VR also argue that these tumours are larger with worse prognosis because of vessel-wall invasion and higher potential of developing liver metastases. ${ }^{26}$ Several studies have shown that true histologic venous invasion has no impact on survival rates. Yekebas et al. found no statistically significant impact of tumour size, resection margin status and histologic vascular wall invasion on life expectancy. ${ }^{27}$ Tseng and colleagues found no difference in median survival between patients with who did and who did not have histopahtologic evidence of vein invasion. ${ }^{12}$ In our study life expectancy of PHR combined with VR was similar to PHR alone.

Few studies have analyzed the durability of the venous reconstruction or reported on the morbidity associated with graft thrombosis. ${ }^{11,12}$ In these series two types of venous reconstruction were performed. When tumour involvement was limited to the superior mesenteric vein (SPV) or portal vein (PV) such that the splenic vein could be preserved, and vessels could be approximated without tension a primary end-to-end anastomosis was performed.
When tumour involved the SMV-splenic vein confluence, splenic vein ligation was necessary. In the remaining eight procedures interposition graft was needed. Dacron grafts with $10 \mathrm{~mm}$ diameter were used. There was no infection after dacron grafting. Of the 6 thromboses observed all were in the acute setting (less than 30 days), however, none of these six patients died secondary to acute thrombosis.

The literature documenting portal vein graft thrombosis rates is sparse. ${ }^{1,12,28,29}$ DiPerna et al. observed patency rates of $93 \%$ and $90 \%$ at 12 and 24 months, respectively. However, in this series, there were only eight portal vein resections with reconstruction. ${ }^{29}$ Tseng et al. noted occlusion in $6.9 \%$ of portal vein grafts, but specific timing and morbidity were not discussed. ${ }^{12}$ The thrombosis rate in this series was lower than in those previously reported $(4.5 \%)$.

Recommendations for anticoagulation following major venous reconstruction for malignancy have varied. ${ }^{1,30}$ No difference was observed in thrombosis rates when comparing patients receiving therapy and those who did not. ${ }^{1}$ Currently, our approach to patients with SMV-PV involvement is similar to other published series. ${ }^{1}$ Primary endto-end anastomosis is performed in those patients requiring segmental resection if it can be accomplished without tension. In those patients who cannot be reconstructed with primary end-to-end anastomosis, an interposition graft is used, with the synthetic dacron graft being our first preference due to its acceptable results in portal decompression surgery. ${ }^{31}$

Generally, the use of a synthetic graft such as dacron or polytetrafluoroethylene (PTFE) is discouraged because of fear from infection or anastomosis disruption from pancreatic juices, and just a few small reports exist. ${ }^{9,15}$ When portomesenteric vein resection is necessary during PD, primary anastomosis of the portomesenteric veins is always the first choice for reconstruction. However portal vein thrombosis was observed frequently after primary vein anastomosis, for several reasons. The most important is probably the anastomotic tension that may go unrecognized when intestines are returned to their original position after pancreaticoduodenectomy. ${ }^{15}$ Some centres use vein interposition graft harvested from the jugular or renal location. However, additional resection of vein is connected with potentially higher morbidity. Additionally the need for vein resection is often not known until the last stage of resection. Because PV clamping time should be kept to a minimum, the suitability and ready availability of synthetic grafts make 
them a desirable conduit for PVR. Synthetic graft provides the necessary length to bridge any gap between the mesenteric vessels and the PV, thus avoiding tension. ${ }^{15}$ The potential risk of infection has restricted the use of synthetic grafts in PVR. Another disadvantage in this scenario is the potential risk of anastomosis disruption following a pancreatic leak. There were no graft infections or anastomotic leaks in this series. It is interesting to note that ligation of the splenic vein, not only in presenting series wasn't presented with long term complications. ${ }^{12}$

Similar survival times after surgical resection in both groups raises once again the question about which factors independently influence the long term outcome in patients with pancreatic cancer. ${ }^{32}$ Survival after surgical resection is related to several factors: most important seem to be the extent of local invasion of the primary tumour, lymph node involvement, vascular invasion, perineural invasion, cellular differentiation, and uninvolved surgical margins. El Ghazzawy et al. reviewed experience in the US Department of Veterans affairs hospitals from 1987-1991. In the group that underwent surgical resection, perineural invasion, microlymphatic invasion, vascular invasion, or tumour differentiation did not independently influence survival when tumours were controlled for stage. ${ }^{33}$ Exactly which factors are truly independent remains controversial.22,32

\section{Conclusions}

Survival of patients with pancreatic cancer who undergo a resection with reconstruction was comparable to those who have a standard pancreaticoduodenectomy with no added mortality or morbidity. Synthetic graft appeared to be an effective and safe option as an interposition graft for portomesenteric venous reconstruction after pancreaticoduodenectomy.

\section{References}

1. Smoot LR, Christein JD, Farnell MB. Durability of portal venous reconstruc tion following resection during pancreaticodudenectomy. J Gastrointest Surg 2006; 10: 1371-5.

2. Marangoni G, O'Sullivan A, Faraj W, Heaton W, Rela M. Pancreatectomy with synchronous vascular resection-An argument in favor. Surgeon 2012; 10: $102-6$.

3. Katz MH, Pisters PW, Evans DB, Sun CC, Lee JE, Fleming JB, et al. Borderline resectable pancreatic cancer: the importance of this emerging stage of disease. J Am Coll Surg 2008; 206: 833-46.
4. Christians K, Evans DB. Pancreaticoduodenectomy and vascular resection: persistent controversy and current recommendations. Ann Surg Oncol 2009; 16: 789-91.

5. Muller SA, Hartel M, Mehrabi A, Welsch T, Martin DJ, Hinz U, et al. Vascular resection in pancreatic cancer surgery: survival determinants. J Gastrointest Surg 2009; 13: 784-92.

6. Martin RC 2nd, Scoggins CR, Egnatashvili V, Staley CA, McMasters KM, Kooby DA. Arterial and venous resection for pancreatic adenocarcinoma: operative and long-term outcomes. Arch Surg 2009; 144: 154-9.

7. Al-Haddad M, Martin JK, Nguyen J, Pungpapong S, Raimondo M, Woodward $\mathrm{T}$, et al. Vascular resection and reconstruction for pancreatic malignancy: a single center survival study. J Gastrointest Surg 2007; 11: 1168-74.

8. Howard TJ, Villanustre N, Moore SA, DeWitt J, LeBlanc J, Maglinte D, et al Efficacy of venous reconstruction in patients with adenocarcinoma of the pancreatic head. J Gastrointest Surg 2003; 7: 1089-95.

9. Chu CK, Farnell MB, Nguyen JH, Stauffer JA, Kooby DA, Sclabas GM, et al. Prosthetic Graft Reconstruction after Portal Vein Resection in Pancreaticoduodenectomy: A Multicenter Analysis. J Am Coll Surg 2010; 211: 316-24.

10. Lai CSE. Vascular resection and reconstruction at pancreatico-duodenectomy: technical issues. Hepatobiliary Pancreat Dis Int 2012; 11: 234-42.

11. Wang F, Arianayagam R, Gill A, Puttaswamy V, Neale M, Gananadha S, et al. Grafts for mesenterico-portal vein resections can be avoided during pancreatoduodenectomy. J Am Coll Surg 2012; 215: 569-79.

12. Tseng JF, Raut CP, Lee JE, Pisters PW, Vauthey JN, Abdalla EK, et al. Pancreaticoduodenectomy with vascular resection: margin status and survival duration. J Gastrointest Surg 2004; 8: 935-50.

13. Kim SH, Min SK, Park D, Min SI, Jang JY, Kim SW, et al. Reconstruction of portal vein and superior mesenteric vein after extensive resection for pancreatic cancer. J Korean Surg Soc 2013; 84: 346-52.

14. Siriwardana HPP, Siriwardena AK. Systematic review of outcome of synchronous portal-superiormesenteric vein resection during pancreatectomy for cancer. Brit J Surg 2006; 93: 662-73.

15. Stauffer JA, Dougherty MK, Kim GP, Nguyen JH. Interposition graft with polytetrafluoroethylene for mesenteric and portal vein reconstruction after pancreaticoduodenectomy. Brit J Surg 2009; 96: 247-52.

16. Dripps RD. New classification of physical status. Anesthesiology 1963; 24: 111.

17. Christians KK, Tsai S, Tolat PP, Evans DB. Critical steps for pancreaticoduodenectomy in the setting of pancreatic adenocarcinoma. J Surg Oncol 2013; 107: 33-8.

18. Rose JB, Rocha F, Alseidi A, Helton S. Posterior 'superior mesenteric artery first' approach for resection of locally advanced pancreatic cancer. Ann Surg Oncol 2014; 21: 1927-8.

19. Malleo G, Pulvirenti A, Marchegiani G, Butturini G, Salvia R, Bassi C. Diagnosis and management of postoperative pancreatic fistula. Langenbecks Arch Surg 2014; 399: 801-10.

20. Clavien PA, Barkun J, de Oliveira ML, Vauthey JN, Dindo D, Schulick RD, et al The Clavien-Dindo classification of surgical complications: five-year experience. Ann Surg 2009; 250: 187-96.

21. Kim WS, Choi DW, Choi SH, Heo JS, Kim MJ, Song SC, et al. Clinical validation of the ISGPF classification and the risk factors of pancreatic fistula formation following duct-to-mucosa pancreaticojejunostomy by one surgeon at a single center. J Gastrointest Surg 2011; 15: 2187-92.

22. Ravikumar R, Sabin C, Hilal MA, Bramhall S, White S, Wigmore S, et al. Portal vein resection in borderline respectable pancreatic cancer: a United Kingdom multicenter study. J Am Coll Surg 2014; 218: 401-11.

23. Zhou Y, Zhang Z, Liu Y, Li B, Xu D. Pancreatectomy combined with superior mesenteric vein - portal vein resection for pancreatic cancer: a meta-analysis. World J Surg 2012; 36: 884-91.

24. Esposito I, Kleef J, Bergmann F, Reiser C, Herpel E, Friess H, et al. Most pancreatic cancer resections are R1 resections. Ann Surg Oncol 2008; 15: 1651-60. 
25. Neoptolemos JP, Stocken DD, Dunn JA, Alomd J, Beger HG, Pederzoli P, et al. Influence of resection margins on survival for patients with pancreatic cancer treated by adjuvant chemoradiation and/or chemotherapy in the ESPAC-1 randomized controlled trial. Ann Surg 2001; 234: 758-68.

26. Ouaissi M, Hubert C, Verhelst R, Astarci P, Sempoux C, Jouret-Mourin A, et al. Vascular reconstruction during pancreaticoduodenectomy for ductal adenocarcinoma of the pancreas improves resectability but does not achieve cure. World J Surg 2010; 34: 2648-61.

27. Yekebas EF, Bogoevski D, Caraldegirmen G, Kunze C, Marx A, Vashist YK, et al. En bloc vascular resection for locally advanced pancreatic malignancies infiltrating major blood vessels: perioperative outcome and long-term survival in 136 patients. Ann Surg 2008; 247: 300-9.

28. Evans DB, Lee JE, Tamm EP, Pisters PW. Pancreaticoduodenectomy (Whipple operation) and total pancreatectomy for cancer. In: Fischer JE, editor Mastery of surgery. Philadelphia: Lippincott Williams and Wilkins; 2007. p. 1299-317.

29. DiPerna CA, Bowdish ME, Weaver FA, Bremner RM, Jabbour N, Skinner D, et al. Concomitant vascular procedures for malignancies with vascular invasion. Arch Surg 2002; 137: 901-7.

30. Quinones-Baldrich WJ, Alktaifi A, Eilber E, Eilber F. Inferior vena cava resection and reconstruction for retroperitoneal tumor excision. J Vasc Surg 2012; 55: 1386-93.

31. Graziano JL, Sullivan HJ. Portal decompression: clinical experience with the "H" graft. Ann Surg 1973; 178: 209-14.

32. Lazaryan A, Kalmadi S, Almhanna K, Pelley R, Kim R. Predictors of clinical outcomes of resected ampullary adenocarcinoma: a single-institution experience. Eur J Surg Oncol 2011; 37: 791-7.

33. el-Ghazzawy AG, Wade TP, Virgo KS, Johnson FE. Recent experience with cancer of the ampulla of Vater in a national hospital group. Am Surg 1995; 61: 607-11. 